\title{
Introducing Biomedical Microsystems into the Electrical Engineering Curriculum
}

\author{
Ian Papautsky and Erik T. K. Peterson \\ Department of Electrical and Computer Engineering and Computer Science \\ Univerrsity of Cincinnati
}

\begin{abstract}
Micromachining or microelectromechanical systems (MEMS) technologies are considered an enabling technology with revolutionary impact on many areas of science and engineering. MEMS technologies are now being applied to health monitoring, diagnosis and therapeutic applications, which is frequently referred to as BioMEMS or Biomedical Microsystems. Biomedical microsystems research includes biological, biomedical, biochemical, and pharmaceutical applications using MEMS-based microsensors and microsystems. To expose our undergraduate seniors and first-year graduate students to the emerging area of Biomedical Microsystems, a course was offered at the University of Cincinnati. The course focused on the basic principles of MEMS and microsensors and their applications in biology and medicine. Topics covered included biochips and lab-on-a-chip devices, microfluidics, biosensors, material biocompatibility, cell/tissue engineering, and point-of-care medicine, including discussions of commercially-available systems. The course was received very well, with enrollment of 38 students from multiple programs in the College of Engineering at University of Cincinnati
\end{abstract}

\section{Introduction}

The life sciences recently experienced a revolution similar to the changes society experienced when the integrated circuit was introduced. The application of microelectromechanical systems (MEMS) technologies in research has already increased the performance of conventional methods in microorganism detection in environmental monitoring, drug discovery in the pharmaceutical industry, and clinical diagnostics. Even more importantly, it is enabling access to new information and applications on the molecular level. ${ }^{1}$ The conceptual paper published in $1990^{2}$ triggered an avalanche of developments and discoveries, which led to an exponential growth of the microfluidics field. ${ }^{3,4}$ As microsystem technologies often employ techniques developed for the microelectronics industry, microfluidic devices were first fabricated in silicon, and later in glass, using standard photolithography and wet etching to produce planar microchannels. ${ }^{5}$ Polymer microfabrication methods have replaced much of the established silicon and glass-based MEMS fabrication techniques ${ }^{6}$ due to complex fabrication procedures, geometric design restrictions, and costs associated with silicon and glass processes. The major advantages of polymers include a wide range of material characteristics, biochemical compatibility, ease of processing, and lower cost. These characteristics make polymers the most promising substrate materials for applications in life sciences. A number of microfluidic systems have been demonstrated in polymers for biomedical applications, including miniaturized 
electrophoresis chips, drug delivery systems, microfluidic mixers, pumps and valves, devices for cell or protein patterning, and microfluidic switches. ${ }^{7,8}$

Devices with microscale dimensions tend to behave differently than their macroscale counterparts, and the unfamiliar physics involved can require modeling and specialized training. Dozens of universities across the country have recently recruited faculty in the field of microtechnologies, specifically focusing on microfluidics and biomedical microtechnologies (or BioMEMS). These initiatives have brought the excitement of BioMEMS research to graduate studies and research programs in Electrical Engineering. While BioMEMS technologies have dramatically altered biomedical, pharmaceutical, and environmental research, they are yet to be successfully transferred to the undergraduate curricula.

\section{The Course}

To introduce electrical engineers to the emerging area of BioMEMS, the authors are developing a course currently entitled "Introduction to Biomedical Microsystems." The objective of the course is to expose students to biomedical microsystems and to teach them fundamental principles of MEMS applications in biology and medicine. Topics covered include BioMEMS fabrication, microsensors for medical applications, biochips and lab-on-a-chip (LOC) devices, microfluidics, biosensors, material biocompatibility, and cell/tissue engineering. These topics are listed in Table 1. Discussion of commercially-available systems is included as well.

The course was designed to be a ten-week long, three-hour course consisting of twenty 75 min lectures. As a 600-level course it was dual-level, intended for the undergraduate seniors and first year graduate students in the Electrical Engineering program. Thus, the course had no prerequisites other than senior class standing. Further, no background in integrated circuit fabrication, MEMS, biomedical instrumentation, or any other specialized area was assumed or required.

Table 1. Topics covered in the "Introduction to Biomedical Microsystems” course.

\begin{tabular}{|c|c|}
\hline Week & Topic \\
\hline 1 & Applications of MEMS in Biology and Medicine \\
\hline 2 & $\begin{array}{l}\text { Fundamentals of microfabrication technologies } \\
\text { for biomedical microsystems }\end{array}$ \\
\hline 3 & Working principles of pressure sensors \\
\hline 4 & Pressure microsensors for clinical applications \\
\hline 5 & Principles of microscale fluid flows \\
\hline 6 & Microfluidic systems: valves, pumps and mixers \\
\hline 7 & Biochips and Lab-on-a-Chip (LOC) devices \\
\hline 8 & Chemical sensors and biosensors \\
\hline \multirow[t]{2}{*}{9} & Biocompatibility \\
\hline & Microsystems for cell engineering \\
\hline 10 & Packaging of biomedical microsystems \\
\hline
\end{tabular}


Topics and their sequence were selected with three criteria in mind. The first was to introduce students to MEMS fabrication. For many, this course was the first exposure to microfabrication and MEMS. Thus, the first two weeks of the course focused on introducing the major techniques and approaches in MEMS fabrication, but with focus on biomedical applications. In those two weeks students learned how to fabricate two fundamental structures that were revisited throughout the rest of the course: a suspended diaphragm and a sealed microchannel. The diaphragm was revisited later in the course when discussing pressure microsensors, and again when discussing microscale valves and pumps. Microchannels were revisited when discussing microfluidics, micromixers, bio/chemical sensors, and biochips. The discussion of MEMS fabrication, and specifically the two fundamental structures, provided students with a common starting point.

The second criterion was not to overwhelm the electrical engineering students by the breadth of the interdisciplinary topics encompassed in BioMEMS. By senior year, the students are knowledgeable in electrical engineering topics, but have few courses outside the department. A demographic survey on the first day of class showed that roughly two thirds of the class had more than 15 courses in electrical engineering, while less than $25 \%$ had fewer than 10 courses. At the same time, two thirds of the students had not taken a single college-level biology course, and almost half the class had not taken any courses in mechanics. Thus, course topics were chosen to build on the students' electrical engineering background earlier in the course and to guide them in the directions of the more mechanically and biologically intense nature of BioMEMS towards the end of the course. Based on this criterion, we chose to discuss a conventional pressure microsensor early in the course and transitioned to biosensors and cell engineering toward the end of the course.

The third and final criterion was to give students an equal exposure to the theoretical principles and their bio/medical applications. For example, after introducing fundamentals of pressure microsensor function, application to intravascular pressure measurements was discussed. This theme of "from theory to application" was carried out throughout the course.

The course used an existing MEMS textbook ${ }^{9}$ intended for a senior or graduate level engineering audience. The textbook was selected due to its focus on microsystem design as well as MEMS fundaments (e.g., fabrication, mechanics, thermodynamics, etc.). At the present time, there is no upper-level undergraduate BioMEMS textbook. The teaching style also included the use of PowerPoint presentations and a whiteboard. To supplement these materials, students were given journal articles to read related to the topics covered in class during that week. The strategy here was to expose students to the state-of-the-art and give them a flavor of what happens in the research environment. The first few articles were review papers. However, by the end of the course, students were reading current research articles.

Students were evaluated through weekly homework assignments, a midterm exam, and a comprehensive final exam. Each homework included three questions: one pertaining to the material covered in class, one pertaining to the material in the assigned textbook reading, and one pertaining to the research article of the week. As the assigned articles changed from reviews to current research papers, questions on homeworks changed appropriately. For review articles, questions aimed to demonstrate knowledge and comprehension, such as questions beginning 
with summarize or describe or compare. Later in the course, students were asked analysis and synthesis style questions, such as those beginning with recommend or explain or what if. Students were also required to perform calculations using the principles of mechanics and microfluidics discussed in class or the research papers. Representative questions are given below.

1. From the paper by Richards-Grayson et al., ${ }^{10}$ what is the appeal for applying MEMS technologies to biology and medicine? Give examples of two specific areas where MEMS technologies are being applied to biological and medical research. In your answer, describe details of the MEMS device(s) used.

2. Based on the papers by G. Stemme and coworkers, ${ }^{11,12}$

(a) Explain how viscosity of the fluid effects the pumping flow rate for a given diaphragm frequency.

(b) According to the data presented, how does the geometry of the diffuser (length and angle) affect the pump performance?

(c) How would the flow rate be affected if the rounded corners at one end (w1) of the diffuser and sharp corners at the other end (w2) were reversed?

(d) Why is the diffuser pump design better for pumping cell suspensions than a conventional diaphragm pump with check valves?

3. From the guest lecture on pressure sensors for clinical applications,

(a) A pressure sensor is characterized by a linear relationship between pressure and voltage where the slope of this curve is the sensitivity. What changes would you expect to see in the sensor's performance if a blood clot were to form over the sensor's surface?

(b) What are the advantages of placing a pressure sensor on a catheter rather than on a guide wire or some alternative delivery vehicle?

(c) Draw a Wheatstone bridge configuration of the pressure sensor's piezoresistors and explain its benefits in signal conditioning.

4. You need to design and fabricate a microfluidic lab-on-a-chip (LOC) for detecting pathogens in blood. The device will have a single microfluidic channel. A blood sample will be introduced at one end of the microchannel and pathogens will be detected using fluorescence at the other end.

(a) You are considering using silicon, glass, or polymethlymethacralate (PMMA) to fabricate the channels. List advantages and disadvantages for each material in the given application.

(b) You decide to fabricate your microchannel in silicon using anisotropic wet etching and capping it with another wafer. List key process steps, in sequence, required to create the sealed microchannel. Assume you start with a bare silicon wafer and you have all of the appropriate masks. Draw a representative cross-section for each step described.

(c) In the fabrication process you created a $2 \mathrm{~cm}$ long microchannel. You experimentally measure the pressure drop along the microchannel to be approximately $150 \mathrm{~Pa}$. However, calculations using Navier-Stokes equations predict a 100 Pa pressure drop. Explain why you observed a higher pressure drop in the channel. Recommend changes you can make to your channel design to reduce the pressure drop. 
5. You are designing a BioMEMS chemical sensor to be implanted in an artery to monitor oxygen concentrations. After making the electrodes, you dip-coat them in silicone to create a thin membrane over the sensor, which you believe is fairly biocompatible.

(a) What types of tests would you need to perform in vitro before implanting the device in a live animal for further testing?

(b) After 2 days of use in vivo, you find that the sensor does not respond to changes in oxygen concentration. All electrical connections seem fine. What is the most likely reason the implanted sensor has stopped responding? What would you change to fix this problem in your next design?

(c) Recommend an appropriate sterilization method and justify your choice.

6. You are fabricating a chip to rapidly screen for pathogens in blood. The device will be fabricated by etching a 20- $\mu$ m deep trench in a glass wafer using HF and capping it with a silicon wafer.

(a) In the space below, outline steps needed to fabricate the described chip. For each step, draw a cross-sectional diagram and label materials.

(b) List five methods you can use to propel fluids through the microchannel and rank them in terms of power and manufacturing ease.

(c) You are considering integrating valves for routing fluids as part of the device in the next revision. List three advantages and three disadvantages of passive valves over their active counterparts.

(d) What biocompatibility issues, if any, do you need to consider? Is your chip biocompatible? Recommend design changes that would improve biocompatibility.

An interactive Web-based Blackboard e-Education platform operated by the University of Cincinnati was used to host the course materials (e.g., PowerPoint slides, research papers, homework assignments). The system permitted quick and effective communication with students in the class through e-mail and announcements, and provided students with feedback on their homework assignments and exams.

Thirty eight students, 26 undergraduate and 12 graduate, representing four programs, including electrical engineering, computer engineering, biomedical engineering, and mechanical engineering enrolled in the course. The class had four female students. This is a small number (approximately 10\% of the class), but is representative of the undergraduate electrical engineering program at the University of Cincinnati.

\section{Course Evaluations}

On the last day of the course, students were provided with an anonymous course evaluation that asked a number of open ended questions. Thirty two students responded to the survey. A detailed discussion of the results will be presented elsewhere, but the most relevant responses are summarized herein. 
In response to the question, "Where the objectives of the course met?" students responded:

- Yes, practical examples were used throughout the course and we could see how the concepts were involved in real world

- Yes, but more applications should be included

- Yes, the goals were met, but specific biology material was not as complete as it could be

To the question, "What was the best aspect of the course?" students responded:

- The material presented was current and on the cutting edge

- Reading journal articles for homework

- The real world examples used throughout the class; it helped to reinforce the material

- The fundamentals were explained clearly which allowed to understand practical applications

To the question, "What would you suggest improving?" students responded:

- Too much information in such a short amount of time; more explanation of fewer topics instead

- The amount of information covered was too great; cutting some of the course martial and covering it in another class in MEMS would have helped in some places where we were rushed

\section{Conclusions}

The assessment revealed that student reaction to the course was generally favorable, although mixed. Most of the students felt that the goals of the course were met. The use of research articles to supplement lecture material worked very well and students were enthusiastic about reading the articles as they provided a real world perspective. This was a new experience for most of the students, and was quite different from what they have come to expect from other senior /first-year graduate level courses. Students also liked the real world examples and applications used to reinforce the lecture material. Thus, the course theme of "from theory to application” was quite successful.

Many students wanted to see more biology and chemistry, including more in-depth coverage of biological applications. Some students felt that too much time was dedicated to the discussion of microfluidics, and that shortening the topic would allow for more discussion of biology and chemistry. However, we feel that the coverage of microfluidics was appropriate, given its importance in operation of biochips and LOCs which are an integral part of many Biomedical Microsystems.

Graduate and undergraduate students had different opinions of the breadth and depth of the course. Graduate students felt that the course did not cover the topics in enough depth. Most of them were enthusiastic about the assigned research articles and thought there should have been more. At the same time, undergraduate students felt overwhelmed by the amount of the material covered in the course. They felt that the pace of the course was too fast for covering this much 
new material. We believe the reason for this perception stems mainly from the interdisciplinary nature of the course. The emerging field of Biomedical Microsystems or BioMEMS is a synthesis of prior knowledge and therefore requires familiarity with other fields. However, complete mastery of those fields is not reasonable. Ideally, other introductory courses in biology and mechanics would cover the fundaments, which would permit a BioMEMS course like the one described herein to cover either more material or perhaps more advanced topics in greater depth. Indeed, such introductory courses are available through other departments at the University of Cincinnati. However, electrical engineering students already have a full schedule of courses within the discipline and often are not able to enroll in such classes outside the department. Another issue is that most students do not realize the need to take these courses until their senior year when they enroll in advanced elective courses such as the one discussed here. Thus, there is a clear need to convey the necessity for interdisciplinary course work early in an electrical engineering student's educational career.

Overall, the two greatest challenges to introducing biomedical microsystems to electrical engineering students are: 1) successfully integrating research topics into the class room, and 2) developing appropriate background to introduce students to the principles of mechanics, microfluidics and biology.

\section{Acknowledgements}

The authors would like to thank the Department of Electrical and Computer Engineering and Computers Science for supporting these efforts to develop and improve a new course to introduce biomedical microsystems to electrical engineers.

\section{References}

1. A. Manz and H. Becekr, Microsystem Technology in Chemistry and Life Sciences, Springer, Heidelberg, Germany, 1998.

2. A. Manz, N. Graber, and H. M. Widmer, "Miniaturized total chemical analysis systems: a novel concept for chemical sensing," Sensors and Actuators B, pp. vol. 1, 244-248, 1990.

3. S. Latta, “Miniaturization, Parallel Processing Come To Lab Devices,” Scientist, vol. 11, no. 18, pp. 1-7, 1997.

4. M. M. Stephan, "Survival in the microfluidics market," Scientist, vol. 18, no. 11, pp. 38-40, 2004.

5. D. J. Harrison, K. Fluri, K. Seiler, Z. Fan, C. Effenhauser, and A. Manz, Science, vol. 261, pp. 895-897, 1993.

6. H. Becker, U. Heim and O. Rotting, "The fabrication of polymer high aspect ratio structures with hot embossing for microfluidic applications,” Proc. SPIE, vol. 3877, pp. 74-79, 1999.

7. G. T. A. Kovacs, Micromachined Transducers Sourcebook, McGraw-Hill, Boston, MA, 1998.

8. M. J. Madou, Fundamentals of Microfabrication: The Science of Miniaturization, $2^{\text {nd }}$ ed., CRC Press, Boca Raton, FL, 2002.

9. T.-R. Hsu, MEMS \& Microsystems: Design and Manufacture. McGraw-Hill, .Boston, MA, 2002. 
10. A. C. Richards-Grayson, R. S. Shawgo, A. M. Johnson, N. T. Flynn, Y. Li, M. J. Cima, and R. Langer, “A BioMEMS review: MEMS technology for physiologically integrated devices,” Proc. IEEE, vol. 92, no. 1, pp. 6-21, 2004.

11. A. Olsson, P. Enoksson, G. Stemme, and E. Stemme, "Micromachined flat-walled valveless diffuser pumps,” $J$. Microelectromech. Syst., vol. 6, no. 2, pp. 161-166, 1997.

12. H. Anderssona, W. van der Wijngaarta, P. Nilssonb, P. Enokssona, G. Stemme, "A valve-less diffuser micropump for microfluidic analytical systems,” Sensors and Actuators B, vol. 72, pp. 259-265, 2001.

\section{Biographical Information}

IAN PAPAUTSKY received his Ph.D. in bioengineering from the University of Utah in 1999. He is currently a tenure-track Assistant Professor of in the Department of Electrical and Computer Engineering and Computer Science at the University of Cincinnati. His research and teaching interests include application of MEMS and microfluidics to biology and medicine.

ERIK T. K. PETERSON received his M.S. in electrical engineering from the University of Cincinnati in 2005, and is currently pursuing his Ph.D. His research interests include microfluidics and MEMS devices for chemical and biological assays. He was the teaching assistant for the Biomedical Microsystems course discussed in this paper. 